\title{
Myotonia in centronuclear myopathy
}

\author{
A. G I L - P E R A L T A, E.RA FEL, J . B A U T IS T A, \\ A N D R. A L B ER C A
}

From the Departments of Neurology and Pathology, Ciudad Sanitaria Virgen del Rocio, Seville, Spain

SUMMARY Centronuclear myopathy, which is unusual because of clinical myotonia, is described in two sisters. The diagnosis was established in adult life, but the first symptoms were noticed in infancy. The outstanding points of the clinical picture were mild amyotrophy, paresis, and clinical myotonia.

Myotubular myopathy (Spiro et al., 1966) is an entity defined by its morphological muscular alterations. The disease displays a notable clinical variability and marked genetic heterogeneity (Radu et al., 1977). Usually it is present early in life, and is found only rarely in adults (Vital et al., 1970). Electrical myotonia (Munsat et al., 1969; Radu et al., 1977) with accompanying cataract has been described in this disease (Hawkes and Absolon, 1975).

We report a family in which two members displayed mild amyotrophy, paresis, and clinical myotonia. Three muscle biopsies performed in two members of the family showed changes typical of centronuclear myopathy. From a clinical point of view, these cases of centronuclear myopathy displayed unusual similarities to myotonic dystrophy. We believe that this is the first report of clinical myotonia in a case of centronuclear myopathy, thus broadening the spectrum of familial muscular diseases accompanied by myotonia, and adding to the masterful classification of Becker (1977).

\section{Case reports}

CASE 1

The proband, a 35 year old woman, had poliomyelitis at one year of age that produced amyotrophy and paresis of the right leg. Since then she has always walked and got up from the floor with difficulty. These facts were attributed by the patient to the sequela of poliomyelitis. At

\footnotetext{
Address for correspondence and reprint requests: Dr E. Rafel, Ciudad Sanitaria Virgen del Rocio, Departamento de Anatomia Patologica, Sevilla - 13, Spain.
}

Accepted 26 May 1978 the age of 27 years she noticed increased muscular difficulties, and needed support to climb stairs. Later on, paresis of the upper extremities, of indeterminate onset, caused difficulty in raising the arms above the shoulders. These symptoms? were not modified by cold weather. The patient repeatedly suffered from corneal ulcers, and within the past year she had noticed macular skin lesions on the right arm.

The patient walked with a waddling gait and $\mathrm{a}$ limp on the right side. Getting up from a prone position was difficult, and accomplished only by climbing up upon herself. There was mild paresis of the orbicularis oculi, flexors of the neck, and sternocleidomastoid muscles. The external ocular muscles were normal. A diffuse paresis of the upper extremities was more pronounced in the proximal muscles, and of lesser degree in the biceps and triceps brachii. The rhomboid major and minor and latissimus dorsi muscles were unaffected. A slight symmetrical amyotrophy of both thenar eminences was seen. Paresis of the truncal flexors and extensor muscles was demonstrated, and the patient exhibited lumbar lordosis. There was bilateral paresis of the lower extremities, with the right side affected more acutely. In the left lower extremity paresis predominated in the proximal musculature and was similar to that observed in the upper extremities. There was mild bilateral retraction of Achilles tendons, more pronounced on the right. The right leg was also shorter. The tendon reflexes were absent, except for the right biceps and left patellar. Percussion myotonia was elicited in the tongue (Fig. 1) and thenar eminences. Mild voluntary myotonia of the hands, which did not change after immersion in $4^{\circ} \mathrm{C}$ cold water for 10 minutes, was recorded. 


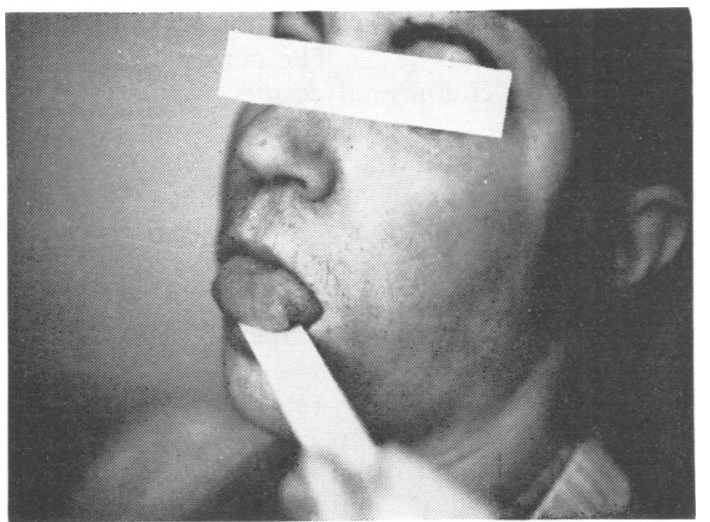

Fig. 1 Myotonia of the tongue.

CASE 2

A 38 year old woman, the sister of the proband, had difficulty in learning to walk, and later required warming-up exercises before being able to walk or run properly. She has always needed help in getting up from the floor and complained of a lack of arm strength. During the winter she suffers from stiffness of the muscles. According to the patient these symptoms have not changed since she was a child.

Physical examination revealed diffuse paresis, mainly proximal in all four extremities. There was a slight talipes cavus and bilateral retraction of the Achilles tendons. Paresis of the truncal extensor and flexor muscles was observed. The flexors of the neck and sternocleidomastoid muscles were unaffected. The external ocular muscles were normal. Tendon reflexes were absent. No muscle wasting was observed. Percussion myotonia of the tongue and mild voluntary myotonia of the hands was induced, and the latter was not affected by immersion in cold water.

\section{FAMILY HISTORY}

Certain facts concerning this family have been established for three generations. The father, who had died at the age of 58 years, was described by his sister and daughters as suffering from muscular stiffness of his hands during cold weather. The mother died at age 64 years, and was described as normal. Nine members from the three generations were examined clinically and found to be normal, including absence of myotonia and cataracts. Two members, one from the second and the other from the third generation, had slight shortening of Achilles tendons. In another sister of the two patients, an EMG was normal.
FURTHER STUDIES

Results of the following studies were normal in both patients: haemoglobin, urinalysis, blood urea, glucose tolerance test, serum electrolytes, serum proteins and immunoelectrophoresis, serology test for syphilis, ASLO, serum phosphatase, transaminase, aldolase, CPK, and LDH, skull and chest radiographs, ECG, and thyroid function studies. Slit lamp examination of the eyes of both patients showed no abnormality. The corneal ulcer of the proband was attributed to probable viral origin. In addition, localised scleroderma of the right arm was diagnosed in the same patient.

\section{EMG studies}

The following muscles were examined in both patients: left deltoid, abductor pollicis brevis, and left tibialis anterior. Slight spontaneous activity, fibrillations, and positive potentials were observed in the abductor pollicis and in the tibialis anterior. Repetitive discharges of high frequency were recorded in all muscles examined, either spontaneously or after muscle percussion; discharges lasted for as long as $\mathbf{2 2}$ seconds with constant frequency and amplitude. There were infrequent discharges of constant frequency and decreasing amplitude lasting up to $1 \frac{1}{2}$ seconds. Rapid interference patterns were present on submaximal contraction. Maximal contraction caused interference patterns of 2-4 mV in amplitude. In the proband's deltoid muscle the mean duration of the motor units was diminished $(43.5 \%)$ with increased polyphasia $(40 \%)$. In the deltoid muscle of the proband's sister the mean duration of the motor units was diminished $(44.5 \%)$ with increased polyphasia $(20 \%)$.

\section{Muscle histology}

Biopsies of the deltoid and biceps brachii muscles in the proband and of the deltoid muscle of the sister were performed. One fragment of the biopsy samples was processed in a routine fashion. A second fragment was frozen, and sections were stained with haematoxylin and eosin ( $\mathrm{H}$ and $\mathrm{E})$ and periodic acid Schiff (PAS). Serial sections were used to demonstrate ATPase (pH 9,4;4,6; 4,3) NADH-D, and SDH activities. The interpretation of the findings and the preparation of the histograms were as described by Dubowitz and Brooke (1973). For electron microscopy a third fragment was fixed in $4 \%$ glutaraldehyde buffered with $0.1 \mathrm{M}$ sodium cacodylate for 24 hours, postfixed in $1 \%$ osmium tetroxide buffered in S-collidine for one hour, dehydrated in graded alcohols and embedded in Epon 812. Thin sections stained with 
uranyl acetate and lead citrate were examined under a Philips 300 electron microscope.

\section{Light microscopy}

In all three muscles the findings were similar. Based on the diameter of the fibres, two sizes, one large and the other small, were seen throughout the transverse sections of the biopsy specimens. Occasionally fibres of intermediate size were also present. In both fibre populations, the nuclei were located exactly in the centre (Fig. 2). With serial sections it was demonstrated that this occurred throughout all fibres. Subsarcolemmic nuclei could be distinguished only rarely among the small fibres. In the centre of some fibres several grouped nuclei, numbering up to four, were present. Chains of centrally located nuclei with free internuclear spaces were found in the longitudinal sections. In the transverse sections stained with $\mathrm{H}$ and $\mathrm{E}$ and in the semi-thin sections from Epon stained with toluidine blue, the central portions of

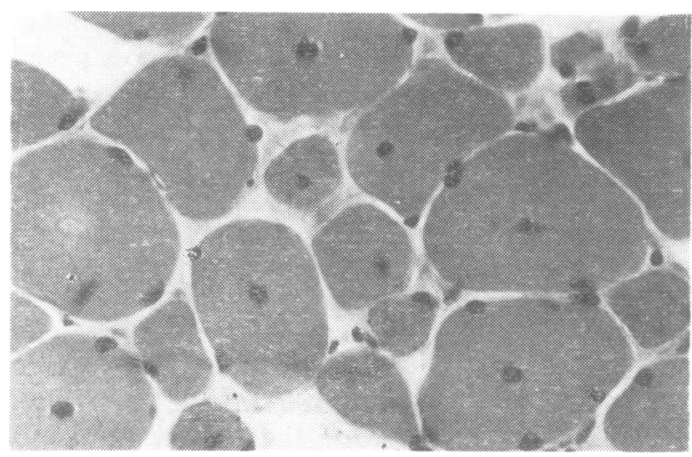

Fig. 2 Transverse section showing small and large fibres with central nuclei. Cryostat section. $H$ and $E$, original magnification $\times 400$.

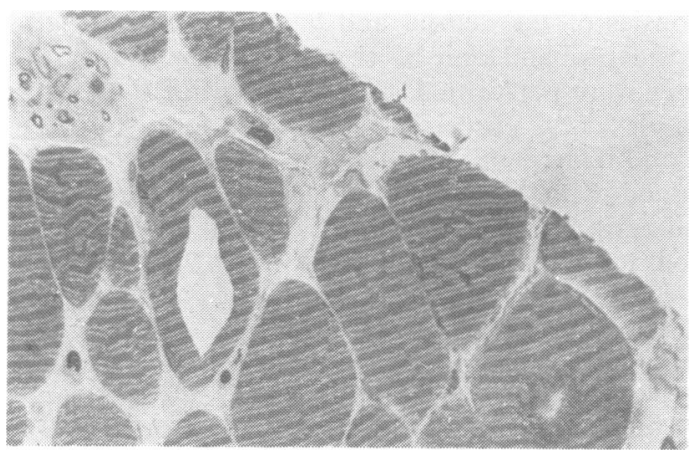

Fig. 3 A fuzzy appearance is seen (lower right) at the centre of the fibre. A large vacuole occupying most of the fibre is noticeable. Epon thick section. Toluidine blue, original magnification $\times 120$. the fibres occasionally showed large vacuoles and a fuzzy appearance (Fig. 3). The perymysial adipose tissue and the endomysial connective tissue were increased.

\section{Histochemical studies}

An abnormal distribution of the oxidative activity was noticeable (Fig. 4) as (a) small calibre fibres in which the activity was scanty in the centre and intense in the periphery, (b) larger fibres where the central activity was absent and the remainder displayed normal intensity, (c) fibres in which the activity was absent in the centre and intense in the peripheral zone, (d) fibres with intense activity in the centre and normal in the periphery, (e) fibres with homogeneous activity throughout, and (f) - fibres with intense activity distributed in a reticular or radial fashion. Similar appearances were found in the sections stained with PAS.

No differentiation of type 1 and 2 fibres was obtained with the oxidative techniques. With the ATPases, the differentiation could only be obtained in a small area of the specimen from the proband's biceps brachii. In this differentiated area the type 1 fibre displayed unusually intense activity at $\mathrm{pH} 9,4$, and the type 2 at all $\mathrm{pH}$ levels showed an activity usually found only in normal type 2a fibres. All fibres showed an absence of activity at their centre.

The mean diameter of the proband's deltoid muscle fibres was $30.90 \mu \mathrm{m}$ with standard deviation of 10.3 and range between 10 and $67 \mu \mathrm{m}$. In the sister, the mean fibre diameter of the same muscle was $41.3 \mu \mathrm{m}$ with standard deviation of 13.18 and a range between 8 and $68 \mu \mathrm{m}$. In the differentiated zone, mentioned above, the type 1 fibre had a mean diameter of $32.47 \mu \mathrm{m}$ with a standard deviation of 13.3 and a range between 8 and $68 \mu \mathrm{m}$. The type 2 fibres had a mean diameter of

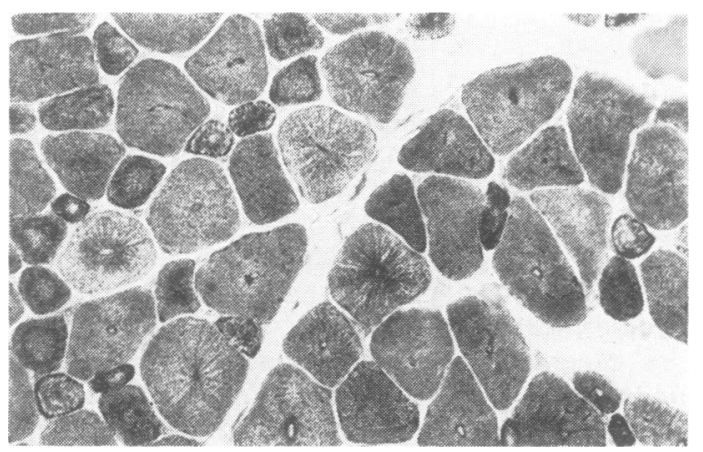

Fig. 4 Transverse section showing a peculiar abnormal distribution of the oxidative activity, Cryostat, NADH-D, original magnification $\times 150$. 


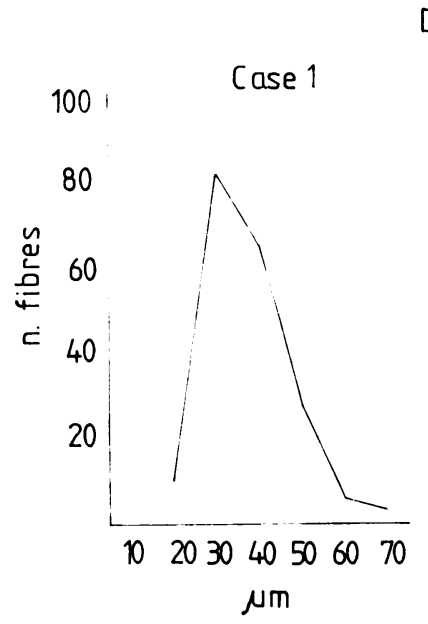

\section{DELTOID}

\section{Case 2}

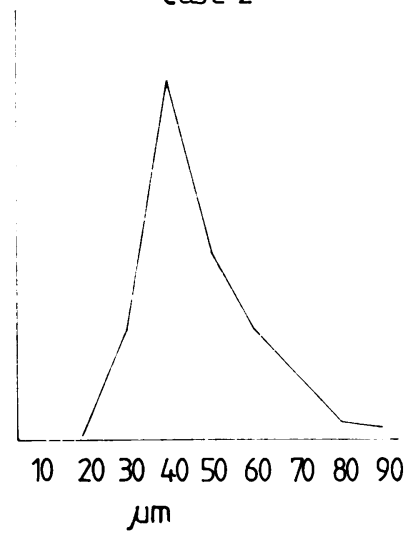

Fig. 5 Histograms of the deltoid muscles in both patients showing atrophic values.
$55.14 \mu \mathrm{m}$ with standard deviation of 10.40 and a range between 30 and $80 \mu \mathrm{m}$. The type 1 fibres represented $76.5 \%$ of the total amount. The histograms of the undifferentiated portions (Fig. 5) demonstrated a deviation towards values typical of atrophy. In the differentiated areas the lower values corresponded to the type 1 fibres, with normal limits and distribution for type 2 fibres (Fig. 6).

\section{Electron microscopy}

The changes observed were similar in both cases.

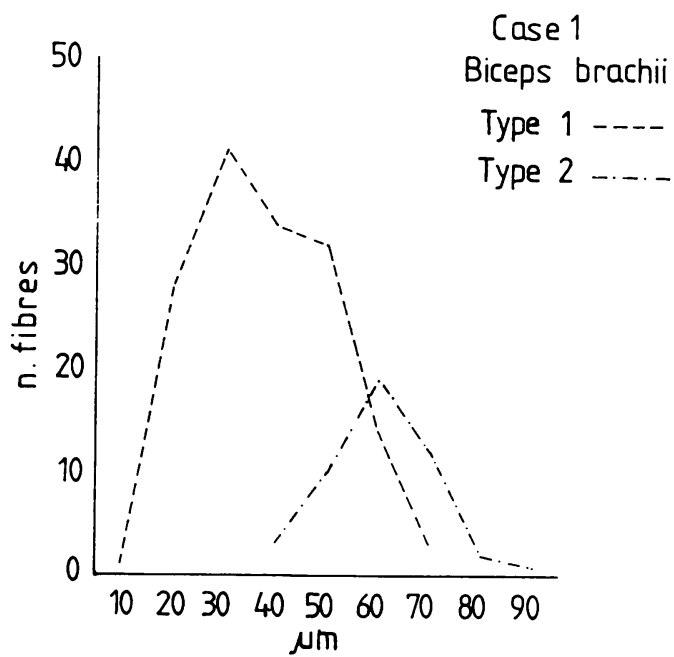

Fig. 6 Histograms of the differentiation zone with atrophic values for type 1 fibres and normal for type 2 fibres.
In most fibres, centrally located nuclei were seen which were normal in shape or slightly indented. Around the nuclei, especially at their poles, and along the centre of the fibre, normal mitochondria, lipid droplets, vacuoles, endoplasmic reticulum, and moderate amounts of glycogen were seen (Fig. 7). In the perinuclear zone the normal banding pattern was altered. Nuclei in chains and nuclei grouped together in the centre of the fibre could also be seen. Sometimes the central areas of the fibres were occupied by granules, possibly of glucogen, and in other fibres large vacuoles limited by membranes were visible. Large deposits of lipids were also present. "Streaming" of $\mathrm{Z}$ bands was rarely seen. The remainder of the structures were well preserved.

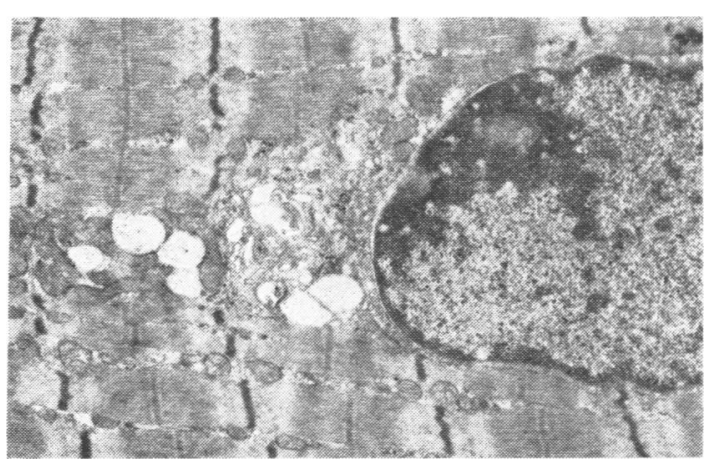

Fig. 7 Central nucleus with abnormal deposit of organelles and vacuoles at one of the poles and normal structure of the myofibrils, original magnification $\times 10000$. 


\section{Discussion}

The presence of muscular paresis, mild amyotrophy, and percussion myotonia in our patients presented a difficult differential diagnosis with myotonic dystrophy. However, a more detailed study of the patients revealed features which were rather unusual for myotonic dystrophy. The long evolution of the paresis and its distribution would make it difficult to accept (a) the normality of the sternocleidomastoid muscle in the proband's sister who had the longer history, (b) the absence of myopathic facies, and (c) the lack of multisystemic involvement, especially cataracts. Furthermore, the onset of the disease in the lower extremities and the mainly proximal distribution would be uncommon in myotonic dystrophy. The age and manner of onset related by one of the patients resembled that of autosomal recessive nondystrophic myotonia. However, the complete clinical feature of this disease is very different (Becker, 1977). The morphological alterations in our cases are diagnostic of centronuclear myopathy (Sher et al., 1967; Campbell et al., 1969; Harriman and Haleem, 1972; Radu et al., 1977).

In centronuclear myopathy fibre type differentiation with ATPase activity has been reported to be abnormal (Goulon et al., 1976; Radu et al., 1977). In one case the abnormality of the ATPase activity of the muscle fibres was shown by the lack of differentiation in types 1 and 2 fibres (Harriman and Haleem, 1972), although the presence of an artefact has not been ruled out. However, the abnormal distribution of the oxidative activity, evident in our case study as well as in another published report (Radu et al., 1977), favours the interpretation of actual absence of differentiation. In our cases, when differentiation of fibre types with ATPase activity at various pH levels was achieved, this only occurred in a very small part of one of the samples. Within this differentiated zone, at ATPase $\mathrm{pH} 9.4$ the type 1 fibres ware darker than usually seen. These facts are in agreement with the theory of defective maturation of the muscle fibres (Spiro et al., 1966; Radu et al., 1977). An abnormality in the DNA structure has been postulated for this defective maturation (Radu and Wild, 1977). Some authors attribute the defect to an alteration of the peripheral nerves (Bethlem et al., 1969; Pongratz et al., 1975), but results of other studies of the intramuscular nerves and of the motor endplates do not support this theory (Harriman and Haleem, 1972; Coërs et al., 1976; Goulon et al., 1976). In our cases, the intramuscular nerves had a normal ultrastructural appearance.
The central position of the nucleus and the atrophy of type 1 fibres present in certain cases of centronuclear myopathy resembles the so-called atrophy of type 1 fibres with central nuclei (Engel et al., 1968; Brooke and Williamson, 1969; Engel, 1970; Karpati et al., 1970; Askanas and Engel, 1975; Inokuchi et al., 1975). Nevertheless, thesc diseases are distinct entities (Harriman and Haleem, 1972; Goulon et al., 1976). A neurogenic mechanism was proposed by Brooke (1973) to explain type 1 fibre hypotrophy in congenital fibre type disproportion. Inokuchi et al. (1975) propose the same mechanism for the hypotrophy found in atrophy of type 1 fibres with central nuclei. Indeed it is possible that this mechanism is the causative atrophic factor in all three diseases and accounts for some of the similarities. The absence of type 2 fibre hypertrophy in our cases of long duration supports the presence of a maturation defect which inhibits this type of fibre from hypertrophying. It is, therefore, possible that two distinct mechanisms exist, one which explains the hypotrophy of type 1 fibre, and a second which explains the absence of type 2 fibre hypertrophy. This second mechanism might also explain the central abnormalities found in type 2 fibres in our cases.

If the faulty maturation theory is accepted, it is difficult to explain why in certain cases the clinical appearance is delayed until adolescence or adulthood. Given the fact that most of the symptoms are silent and only slightly incapacitate the patient, it is difficult to ascertain the precise time of onset in adult patients. It is possible that in some patients the onset had actually passed unnoticed during their infancy (Vital et al., 1970). This may also explain the discrepancy in the ages of onset in various afflicted members of the same family (McLeod et al., 1972). The family presented here is another clear example of this difficulty, and the problem is compounded by the fact that the proband had earlier suffered from poliomyelitis.

In some cases (Munsat et al., 1969; Hawkes and Absolon, 1975; Radu et al., 1977) bizarre high frequency EMG discharges of myotonic or pseudomyotonic character have been recorded. However, to our knowledge, no cases of clinical percussion or voluntary myotonia have been described in the presence of centronuclear myopathy. The existence of this myotonia could be explained if we accepted the coexisten $s c$ of centronuclear and familial myotonic disease. This is improbable as no myotonic manifestations have been encountered in any other members of the family who are not affected by the centronuclear myopathy. Yet another explanation might be that we are dealing with a myotonic dystrophy which is being mor- 
phologically expressed in an unusual way. To our knowledge, morphological findings of centronuclear myopathy have never been described in relatives of a patient with myotonic dystrophy. Since, in our case study, the three biopsy samples were typical of centronuclear myopathy, it is logical to conclude that the clinical myotonia is actually an expression of this disease.

The genetic alteration causing the myotonic dystrophy may in some way also provoke the appearance of centronuclear myopathy. In muscle samples of severe cases of neonatal myotonic dystrophy (Sarnat and Silbert, 1976) certain morphological features similar to those seen in neonatal cases of recessive sex-linked centronuclear myopathy were found by Barth et al. (1975). However, in none of the biopsies taken at a later date in neonatal myotonic dystrophy (Karpati et al., 1973; Farkas et al., 1974) was the morphology of centronuclear myopathy found.

It seems that our cases are cen:ronuclear myojathies associated with clinical myotonia. Without the verification of a muscle biopsy our two patients could easily have been diagnosed as suffering from myotonic dystrophy with unusual clinical features. Therefore, these cases of centronuclear myopathy resembling myotonic dystrophy encompass the most important characteristic of this study, and it is possible that these cases would previously have been included among the myotonic dystrophies.

We would like to express our appreciation to Dr C. Montero, pathologist, for kindly making the histochemical material available to us, to $\mathrm{Dr}$ J. M. Castilla for the electromyographic studies, and S. Shewchuk, PhD, for his help in preparing the manuscript.

\section{References}

Askanas, V., and Engel, W. K. (1975). Distinct subtypes of type 1 fibers of human skeletal muscle. Neurology (Minneapolis), 25, 879-887.

Barth, P. G., Van Wijngaarden, G. K., and Bethlem, J. (1975). X-linked myotubular myopathy with fatal necnatal asphyxia. Ncurology (Minneapolis), 25, 531-536.

Becker, P. E. (1977). Myotonia congenita and syndromes associated with myotonia. Clinical-genetic studies of the non-dystrophic myotonias. In Topics in Human Genetics, vol. 3, pp. 1-154. Edited by R. E. Becker. Georg Thieme: Stuttgart.

Bethlem, J., Van Wijngaarden, G. K., Meijer, A. E. F. H., and Hülsmann, W. C. (1969). Neuromuscular disease with type 1 fiber atrophy, central nuclei, and myotube-like structures. Neurology (Minneapolis), 19, 705-710.
Brooke, M. H. (1973). Congenital fiber type dysproportion. In Proceedings of the Second International Congress on Muscle Diseases, Perth, Australia, Part 2 Clinical Studies in Myology. Edited by B. A. Kakulas. Excerpta Medica: Amsterdam.

Brooke, M. H., and Williamson, T. (1969). An adult case of type 1 muscle fiber hypotrophy: an abnormality of monosynaptic reflex function. Neurology (Minneapolis), 19, 280.

Campbell, M. J., Rebeiz, J. J., and Walton, J. N. (1969). Myotubular, centronuclear or pericentronuclear myopathy? Journal of the Neurological Sciences, 8, 425-443.

Coërs, C., Teleman-Torpet, N., Gerard, J. M., Szliwowski, H., Bethlem, J., and Van Wijngaarden, G. K. (1976). Changes in motor innervation and histochemical pattern of muscle fibers in some congenital myopathies. Neurology (Minneapolis), 26, 1046-1053.

Dubowitz, V., and Brooke, M. H. (1973). Muscle Biopsy: A Modern Approach. W. B. Saunders: London.

Engel, W. K. (1970). Selective and nonselective susceptibility of muscle fiber types. A new approach to human neuromuscular diseases. Archives of Neurology (Chicago), 22, 97-117.

Engel, W. K., Gold, G. N., and Karpati, G. (1968). Type 1 fiber hypotrophy and central nuclei. Archives of Neurology (Chicago), 18, 435-444.

Farkas, E., Tomé, F. M. S., Fardeau, M., Arsenionunes, M. L., Dreyfus, P., and Diebler, M. F. (1974). Histochemical and ultrastructural study of muscle biopsies in 3 cases of dystrophia myotonica in the newborn child. Journal of the Neurologica! Sciences, 21, 273-788.

Goulon, M., Fardeau, M., Got, Cl., Babinet, P., and Manko, E. (1976). Myopathie centro-nucléaire d'expression clinique tardive. Etude clinique, histologique et ultrastructurale d'une nouvelle observation. Revue Neurologique, 132, 275-290.

Harriman, D. G. F., and Haleem, M. A. (1972). Centronuclear myopathy in old age. Journal of Pathology, 108, 237-247.

Hawkes, C. H., and Absolon, M. J. (1975). Myotubular myopathy associated with cataract and electrical myotonia. Journal of Neurology, Neurosurgery, and Psychiatry, 38, 761-764.

Inokuchi, T., Umezaki, H., and Santa, T. (1975). A case of type 1 muscle fibre hypotrophy and internal nuclei. Journal of Neurology, Neurosurgery, and Psychiatry, 38, 475-482.

Karpati, G., Carpenter, S., and Nelson, R. F. (1970). Type 1 muscle fibre atrophy and central nuclei. A rare familial neuromuscular disease. Journal of the Neurological Sciences, 10, 489-500.

Karpati, G., Carpenter, S., Waters, G. V., Eisen, A. A., and Anderman, F. (1973). Infantile myotonic dystrophy. Histochemical and electron microzcopic features in skeletal muscle. Neurology (Minneapolis), 23, 1066-1077.

McLeod, J. G., Baker, W. D. C., Lethlean, A. K., and Shorey, C. D. (1972). Centronuclear myopathy 
with autosomal dominant inheritance. Journal of the Neurological Sciences, 15, 375-387.

Munsat, T. L., Thompson, L. R., and Coleman, R. F. (1969). Centronuclear ("myotubular") myopathy. Archives of Neurology (Chicago), 20, 120-131.

Pongratz, D., Heuser, M., Mittelbach, F., and Struppler, A. (1975). Die sogenannte congenitale centronucleare Myopathie-eine primare Neuropathie? Acta Neuropathologica (Berlin), 32, 9-19.

Radu, H., and Wild, F. (1977). Abnormal DNA in centronuclear myopathy. Eleventh World Congress of Neurology, pp. 291. Excerpta Medica: Amsterdam.

Radu, H., Killyen, I., Ionescu, V., and Radu, A. (1977). Myotubular (centronuclear) (neuro-) myopathy. I. Clinical, genetical and morphological studies. European Neurology, 15, 285-300.
Sarnat, H. B., and Silbert, S. W. (1976). Maturation arrest of fetal muscle in neonatal myotonic dystrophy. Archives of Neurology (Chicago), 33, 466-474.

Sher, J. H., Rimalovsky, A. B., Athanassiades, T. J., and Aronson, S. M. (1967). Familial centronuclear myopathy: a clinical and pathological study. Neurology (Minneapolis), 17, 727-742.

Spiro, A. J., Shy, G. M., and Gonatas, N. K. (1966). Myotubular myopathy. Archives of Neurology (Chicago), 14, 1-14.

Vital, Cl., Vallat, J. M., Martin, F., Le Blanc, M., and Bergouignan, M. (1970). Etude clinique et ultrastructurale d'un cas de myopathie centronucléaire (myotubular myopathy) de l'adulte. Revue Neurologique, 123, 117-130. 